\title{
Retraction-Related Acute Liver Failure after Urological Laparoscopic Surgery
}

\author{
Tetsuo Nozaki Tomonori Kato Akira Komiya Hideki Fuse \\ Department of Urology, Graduate School of Medicine and Pharmaceutical Sciences for Research, University of Toyama, Toyama, Japan
}

\section{Key Words}

Laparoscopic surgery $\cdot$ Liver injury $\cdot$ Retractor •

Acute liver failure

\begin{abstract}
Liver retraction is necessary for optimal exposure during laparoscopic right renal surgery. We described a patient who developed fulminant liver failure as a result of liver retractor-induced excessive ischemic changes in the right lobe of the liver. A 37-year-old male underwent a right side laparoscopic pyeloplasty for ureteropelvic junction obstruction. At the beginning of the operation, a small snake retractor was placed through a 5-mm port under direct vision. The liver was lifted in the appropriate direction to optimize exposure by using the laparoscope holder. The operation was prolonged. However, we achieved significant improvements in the efficiency of liver retraction using the holder. On the first postoperative day, the patient's serum levels of GOT, GPT and LDH had remarkably increased. A computerized tomogram confirmed the presence of excessive ischemic changes of the right lobe of the liver. Our method which used a laparoscope holder device for liver retraction maintained a better surgical field. However, neglecting to make minor adjustments to the positioning of the retractor can cause significant pressure on the liver parenchyma in a single area. As surgical procedures increase in complexity, the surgeon should keep these potential side effects in mind and shift the retraction point at regular intervals. In this report, we discussed various types of retractor-related liver injuries and their management, and highlighted the importance of intermittent release of retraction during prolonged surgery. Copyright $\odot 2013 \mathrm{~S}$. Karger AG, Basel
\end{abstract}

\section{KARGER}

Fax +4161306 1234

E-Mail karger@karger.ch

www.karger.com
(C) 2013 S. Karger AG, Basel

$1015-9770 / 13 / 0074-0199 \$ 38.00 / 0$

Accessible online at:

www.karger.com/cur

\section{Introduction}

During laparoscopic right adrenal and renal surgery, liver retraction is essential to enable full visualization [1]. However, once the liver is retracted and an adequate view is obtained, the camera lens is mainly focused on the immediate operative field, not on the retracted liver. As a result, surgical assistants are regularly entrusted with the use of an unfamiliar instrument to retract an unseen liver for an extended period of time. As the surgical procedures increase in complexity, it is difficult to constantly maintain the proper amount of isometric force and the proper retractor position to maintain optimal exposure. As a result of muscle fatigue by the assistant surgeon, the retractors may accidentally move, or excessive force may inadvertently be applied when the liver is retracted. Injury to the liver may then occur out of the surgical field of vision during instrument manipulation [2-8]. To overcome these problems, we previously described the use of a laparoscope holder (Karl Storz GmBH and Co., Tuttlingen, Germany) to help facilitate lifting the liver during right adrenal and renal surgery [9]. We have achieved significant improvements in the efficiency of liver retraction by using the laparoscope holder. Furthermore, this tool eliminates assistant muscle fatigue, instrument migration, and the need for a dedicated second terminal assistant. One of the potential advantages of using these passive systems is that the retractor always exerts the same defined pressure without fatigue. However, if the retraction force is excessive or maintained at the same point for a long time, it may lead to ischemic injury to the retracted organ. 


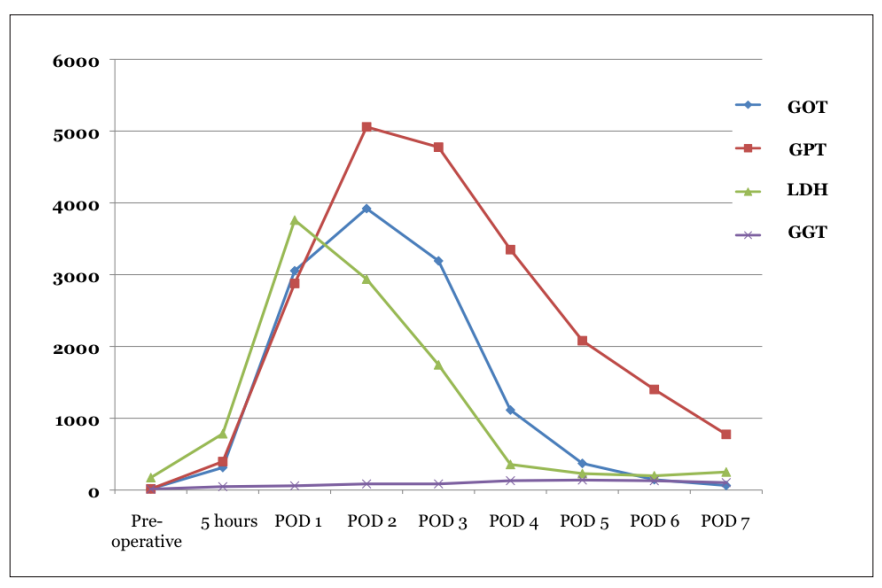

Fig. 1. Temporal changes in the serum GOT level. A value $>70$ times the normal GOT level was reached 48 hours after the operation (GOT normal level; 5-55 IU/L).

We describe a patient who developed acute liver failure (ALF) as a result of prolonged compression of the hepatic pedicle (i.e. the branch of the hepatic artery and portal vein) during laparoscopic right pyeloplasty. Although ALF related to the use of laparoscopic liver retraction is a rare complication, it can have serious implications on patient outcome. We present a case of ALF induced by using the laparoscope holder device for liver retraction, and provide a review of the pertinent literature.

\section{Case Report}

A previously healthy 37-year-old male underwent right side laparoscopic pyeloplasty for ureteropelvic junction obstruction (UPJO). The operation was performed with the patient under general anesthesia with epidural analgesia. Our method using the laparoscope holder device for liver retraction has been described in detail elsewhere [9]. With the patient in the lateral decubitus position, 4 ports were placed transperitoneally. Thereafter, the Flex Arm Surgical Holder (Bookwalter Endoscopic Instrument; Codman, Raynham, MA) was quickly clamped to the table via the base rod over a sterile drape.

The Flex Arm Surgical Holder consists of four components: a table holder, a base rod, an articulating arm, and a precision clamp. The clamp, located at the tip of the holding system, accommodates instruments of various sizes. The articulating arm has three joints and is operated manually to aid in positioning. The laparoscope holder is also fastened, securing the retractor in the desired position. If required, minor repositioning of the device can easily be performed by the assistant. The right lobe of the liver was retracted using a small snake retractor (Snowden Pencer, McGaw Park, IL). The snake retractor was inserted through a 5-mm

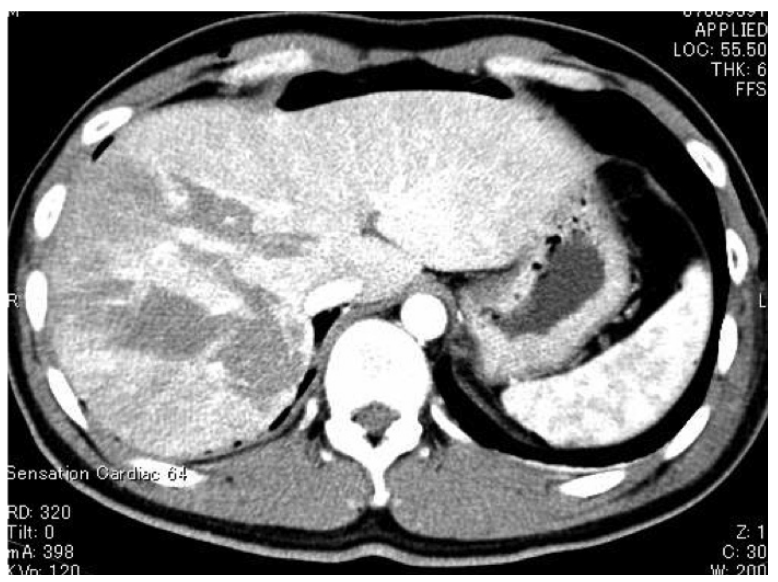

Fig. 2. Dynamic contrast enhanced CT scan images demonstrating excessive ischemic changes of the right lobe of the liver.

trocar just below the tip of the 11th rib, and was placed under the right lobe of the liver, retracting it antero-superiorly to expose the whole right kidney.

The dissection was begun by mobilizing the ascending colon and the duodenum medially by incising along the avascular line of Toldt. After the Gerota fascia was opened, the proximal ureter and ureteropelvic junction were cleared. Because the UPJO was caused by a crossing vein, the vessel was dissected free, and the strictured region was sharply excised. Thereafter, the renal pelvis and ureter were transposed anterior to the vessel. The ureter was spatulated on its lateral aspect, and the redundant renal pelvis was excised.

After the complete laparoscopic mobilization of the UPJ, a standard Anderson-Hynes dismembered pyeloplasty was performed. The anastomosis between the ureter and the renal pelvis was performed with interrupted 4-0 polyglactin sutures. After the completion of the posterior wall anastomosis, an antegrade double-J stent with guidewire was advanced into the ureter and down to the bladder using laparoscopic forceps through the $5 \mathrm{~mm}$ port. Thereafter, the proximal end of the double-J stent was passed into the renal pelvis. Next, the anterior anastomosis was completed with interrupted sutures. The redundant opened renal pelvis was sutured in a running fashion. The peritoneum was closed, a drainage tube was left, and the port site was closed. The total laparoscopic time of the operation was $331 \mathrm{~min}$. During these procedures, we needed continuous liver retraction to obtain effective working space for extensive excision of the redundant pelvis and the excised redundant pelvic closure. Minor repositioning of the laparoscopic liver retractor was performed several times by the assistant.

The patient's serum levels of GOT, GPT and LDH rapidly increased at 5 hours after the operation (GOT: 313 IU/L; GPT: 396 IU/L and LDH: 784 IU/L). At first, we suspected retraction-related liver dysfunction, and $40 \mathrm{ml}$ of neo-minophagen $\mathrm{C}$, a compound mainly composed of glycyrrhizic acid, was intravenously injected. However, on postoperative day 1 , his serum levels of GOT, GPT 
and LDH were found to have further increased (GOT: 3,054 IU/L; GPT: 2,878 IU/L and LDH: 3,760 IU/L) (fig. 1). Because no obvious cause was clinically evident, the patient underwent an urgent dynamic enhanced abdominal computerized tomogram (CT). The CT scan demonstrated extensive liver ischemic changes (fig. 2), and we considered that the occlusion of the right hepatic artery and right portal vein must have simultaneously occurred during surgery. Fortunately, there was no finding of any obstructive signs in the arterial phase, portal thrombosis in the portal venous phase, or gas-filled liver necrosis. We refrained from performing an emergency exploratory laparotomy or thrombolytic therapy.

Regarding the laboratory data, thrombocytopenia, a prolonged prothrombin time, and increased FDP were observed, which fulfilled the diagnostic criteria for disseminated intravascular coagulation (DIC). The administration of nafamostat mesylate at a dose of $200 \mathrm{mg} /$ day was started on postoperative day 1 . After the onset of the liver injury, the laboratory blood test showed impairment of coagulation activity parameters, and the DIC was resolved by postoperative day 4 . The patient was eventually discharged from the hospital on postoperative day 14 without further significant events. A repeat CT scan at the time of discharge revealed an improved appearance of the liver and a reduction of liver ischemic changes. His laboratory data six weeks after surgery showed a complete resolution of liver function.

\section{Discussion}

From a review of the available literature, it was concluded that liver injuries occurring secondary to mechanical retraction of the liver during laparoscopic surgery can be classified into 2 types $[2-8,10]$. The first type of retraction injury is a result of a retraction-related parenchymal fracture or tear, which can occur as a direct injury due to the blade of the retractor $[11,12]$. These injuries and parenchymal fractures are more likely to occur if the liver lobe is enlarged, fatty and/or fragile. Most such parenchymal trauma is identified at the time of its occurrence and only causes bleeding, which is often self-limiting. Infrequently, such direct retraction-related parenchymal injury presents postoperatively due to a slowly developing subcapsular hematoma [8]. Postoperative hemorrhage due to a hematoma or tear of the liver is a rare but life-threatening complication. These complications have been reported during laparoscopic cholecystectomy or adrenalectomy, but have not been reported following open surgery. Therefore, these complications should be recognized as a potential risk associated with the laparoscopic approach. In cases with conventional laparoscopic liver lift by an assistant, the potential disadvantages include over-retraction of tissues, which may lead to liver tears. Although the assistant should bear this in mind and use the retractor with an appropriate amount of force for the liver, it is difficult to constantly maintain

Liver Failure after Urological

Laparoscopic Surgery the proper amount of isometric force and the proper retractor position to maintain optimal exposure due to muscle fatigue, especially during long operations. Therefore, a new method for facilitating liver retraction is needed. During right laparoscopic adrenalectomy (LA), retraction of the liver is essential to obtain a better laparoscopic view. In an attempt to optimize the right LA procedure, Aminsharifi et al. [13] reported the technique of right LA with a modified trocar arrangement and dynamic liver retraction. Compared to the standard technique, this approach can be effectively performed even in challenging cases and can significantly shorten the operative time. Sugimoto et al. [14] suggested that tight attachment between the lower surface of the liver and the right adrenal gland was caused by the intermingling of parenchymal cells of both organs. Surgeons have to keep this issue in mind and pay careful attention during right LA to avoid unnecessary adverse events.

The second type of injury occurs from parenchymal congestion as a result of prolonged compression. It is invariably seen during laparoscopic procedures because the liver parenchyma is trapped by the retractor. Fortunately, such pressure-related injuries are usually temporary and without any major clinical significance. Clinically, they are reflected as a transient asymptomatic elevation of liver enzymes, particularly GOT. This benign phenomenon has been described previously as retraction transaminitis, where the levels remained six times the upper normal limit [15]. However, depending on the duration of retraction and the amount of liver tissue trapped, the congestion can be severe enough to cause parenchymal infarctions. Such injuries are typically identified on postoperative CT scans and may have variable morphological features $[16,17]$. These infarcts vary from wedge-shaped lesions, to rounded lesions, or irregular lesions. The finding of gas in the area of infarction is usually considered to be a sign of infection, which is generally considered an indication for intervention. Tamhankar et al. [10] reported a case of massive liver necrosis secondary to a Nathanson retractor injury after laparoscopic liver retraction. The patient developed a remarkable increase in the serum level of GOT, with a peak level 20 times the upper normal limit, which is much higher than that typically found in cases of transient transaminitidis. Because their CT examination showed gas-filled liver necrosis and the presence of septic shock, a decision was made to perform an exploratory laparotomy. At reoperation, the left lobe of the liver was observed to be necrotic and turgid. A left lateral sectionectomy was performed. The patient remained on inotropic support postoperatively and died 
from a myocardial infarction 14 hours later. Their patient had undergone prolonged retraction with a Nathanson retractor while in the reverse Trendelenburg position, which thus led to compression of the left lobe of the liver between the diaphragm and the retractor blade as a result of the pressures generated by the weight of the patient.

Laparoscopic surgery has rapidly developed and has recently been applied for more complicated surgical procedures. As the surgical procedures increase in complexity, the surgeon needs to concentrate more on the immediate surgical site instead of on maintaining the surgical field. Our method using a laparoscope holder device for organ retraction may in the future become applicable for various types of laparoscopic surgery because it constantly maintains a better surgical field through the proper retractor position and keeps the proper amount of isometric force for optimal exposure $[18,19]$. The incidence of excessive force, instrument migration due to muscle fatigue, and liver injury as a result of improper instrument manipulation are all substantially reduced by using this device. However, these passive organ retraction systems always exert the same defined pressure without fatigue, and are unable to respond to anatomical shifts. Neglecting to perform minor repositioning of the device at regular intervals can thus result in the occurrence of significant pressure on the liver parenchyma in a single area, particularly when the surgical procedure is prolonged. In the present case, our procedural perfor- mance time was prolonged. We were still in the learning curve for laparoscopic pyeloplasty. Several reports have discussed the stiff learning curve of laparoscopic pyeloplasty, because free-hand suturing of the ureteropelvic junction remains technically challenging [20-22]. Moreover, in this case, computed tomography and a retrograde pyelography demonstrated an advanced right hydronephrosis, which necessitated continuous liver retraction to obtain the effective working space. The surgeon should keep these potential side effects in mind, and use the retractor at the appropriate tension for the specific organs, and as surgical procedures increase in complexity, the retraction point should be shifted at regular intervals.

\section{Conclusion}

This is the first reported case of massive liver ischemic changes due to a sustained pressure injury inflicted by using a laparoscope holder device for liver retraction. Laparoscopic surgery is being increasingly performed using such liver retractors, and the necessity of preventing such injuries is often overlooked. This report emphasizes the need for awareness of these injuries and we recommend that the liver retractor should therefore be removed intermittently during prolonged surgery to re-establish hepatic lobe circulation.

\section{References}

1 Paolucci V, Schaeff B, Gutt CN, Litynski GS: Exposure of the operative field in laparoscopic surgery. Surg Endosc 1997;11:856-863.

2 Shamiyeh A, Wayand W: Laparoscopic cholecystectomy: early and late complications and their treatment. Langenbecks Arch Surg 2004:389:164-171.

-3 Antsaklis GI, Karanikas ID, Sakellaridis TE, Alexiou CP, Konstantinidou E, Economou $\mathrm{N}$ : Subcapsular hematoma of the liver: a rare complication of the laparoscopic cholecystectomy. Minerva Chir 2009;64:322-323.

-4 Bhandarkar DS, Katara AN, Shah RS: Intrahepatic subcapsular hematoma complicating laparoscopic cholecystectomy. Surg Endosc 2004;18:868-870.

5 Shetty GS, Falconer JS, Benyounes H: Subcapsular hematoma of the liver after laparoscopic cholecystectomy. J Laparoendosc Adv Surg Tech A 2005;15:48-50.
6 Vuilleumier H, Halkic N: Laparoscopic cholecystectomy as a day surgery procedure: implementation and audit of 136 consecutive cases in a university hospital. World J Surg 2004;28:737-740.

7 Shibuya K, Midorikawa Y, Mushiake H, Watanabe M, Yamakawa T, Sugiyama Y: Ruptured hepatic subcapsular hematoma following laparoscopic cholecystectomy: report of a case. Biosci Trends 2010;4:355-358.

$>8$ Yoon GH, Dunn MD: Case report: subcapsular hepatic hematoma: retraction injury during laparoscopic adrenalectomy. J Endourol 2006;20:127-129.

-9 Nozaki T, Iida H, Morii A, Fujiuchi Y, Komiya A, Fuse H: Use of the laparoscope holder for liver retraction during urological laparoscopic surgery. Curr Urol 2012;6:99-101.

10 Tamhankar AP, Kelty CJ, Jacob G: Retraction-related liver lobe necrosis after laparoscopic gastric surgery. JSLS 2011;15:117121
11 Hunter JG, Trus TL, Branum GD, Waring JP, Wood WC: A physiologic approach to laparoscopic fundoplication for gastroesophageal reflux disease. Ann Surg 1996;223:673-685.

$\checkmark 12$ Medina LT, Veintimilla R, Williams MD, Fenoglio ME: Laparoscopic fundoplication. J Laparoendosc Surg 1996;6:219-226.

-13 Aminsharifi A, Mohammadian R, Niroomand $\mathrm{R}$, Afsar F: Optimizing the technique of right laparoscopic adrenalectomy with a modified trocar arrangement and dynamic liver retraction: A comparative study with standard technique. Int J Surg 2013;11:463-466.

-14 Sugimoto M, Hirama H, Hayashida Y, Shibuya S, Ueda N, Kakehi Y: Cellular intermingling between adrenal gland and liver: an infrequent cause of incomplete resection at right adrenalectomy. J Endourol 2013;27: 804-808. 
15 Morris-Stiff G, Jones R, Mitchell S, Barton K, Hassn A: Retraction transaminitis: an inevitable but benign complication of laparoscopic fundoplication. World J Surg 2008;32: 2650-2654.

16 Yu J, Turner MA, Cho SR, Fulcher AS, DeMaria EJ, Kellum JM, Sugerman HJ: Normal anatomy and complications after gastric bypass surgery: helical CT findings. Radiology 2004;231:753-760.
17 Stewart BG, Gervais DA, O’Neill MJ, Boland GW, Hahn PF, Mueller PR: Imaging and percutaneous treatment of secondarily infected hepatic infarctions. AJR Am J Roentgenol 2008;190:601-607.

18 Zorn KC, Gofrit ON, Zagaja GP, Shalhav AL: Use of the endoholder device during robotic-assisted laparoscopic radical prostatectomy: the "poor man's" fourth arm equivalent. J Endourol 2008;22:385-388.

-19 Landman J, Venkatesh R, Vanlangendonck R, Andriole GL: Application of a fixed retractor system to facilitate laparoscopic radical prostatectomy. J Urol 2004;171:783-785.
20 Inagaki T, Rha KH, Ong AM, Kavoussi LR, Jarrett TW: Laparoscopic pyeloplasty: current status. BJU Int 2005;95(suppl 2):102105.

-21 Sorensen MD, Delostrinos C, Johnson MH, Grady RW, Lendvay TS: Comparison of the learning curve and outcomes of robotic assisted pediatric pyeloplasty. J Urol 2011;185(6 suppl):2517-2522.

-22 Mandhani A, Kumar D, Kumar A, Dubey D, Kapoor R: Steps to reduce operative time in laparoscopic dismembered pyeloplasty for moderate to large renal pelvis. Urology 2005; 66:981-984. 